\title{
Efeitos de variáveis individuais e contextuais sobre desempenho individual no trabalho
}

\author{
Francisco Antonio Coelho Junior \\ Jairo Eduardo Borges-Andrade \\ Universidade de Brasília
}

\begin{abstract}
Resumo
O presente trabalho objetivou testar empiricamente um modelo teórico multinível de predição de desempenho individual no trabalho. As variáveis antecedentes investigadas, isoladas ou em interação, corresponderam à idade, gênero, cargo, grau de escolaridade, tempo de serviço e percepção de suporte à aprendizagem, medidas no nível individual e de contexto, e satisfação no trabalho, de nível individual. A pesquisa foi realizada em uma empresa pública, do ramo de pesquisas agropecuária e atuação nacional. A amostra $(N=808)$ contou com funcionários distribuídos em 45 unidades centralizadas e descentralizadas da empresa pelo Brasil. A coleta de dados foi realizada à distância, via e-mail. Os resultados multiníveis corroboraram o modelo teórico de pesquisa hipotetizado e evidenciaram que a variância de desempenho foi explicada por distintos preditores de nível individual e de contexto, isoladamente ou em interação.
\end{abstract}

Palavras-chave: desempenho no trabalho; satisfação no trabalho; suporte à aprendizagem; regressão multinível.

\begin{abstract}
Effects of individuals and contextual variables on individual performance at work. This paper aims to empirically test a theoretical multilevel model for the prediction of individual performance at work. Antecedent variables, isolated or in interaction, were age, gender, function, scholarity, period of function and perception of learning support, in the individual and contextual levels, and satisfaction at work, an individual variable. This study was accomplished in a public corporation which deals with agricultural research in a national scope. The participants $(N=808)$ were distribute in 45 central and noncentral units for the Brazil. Data collection was done online, by e-mail. The multilevel results confirm the hypothetic theoretical model and make evident that the performance's variance were predicted by different individuals and context variables, isolated or in interaction.
\end{abstract}

Keywords: work performance; work satisfaction; learning support; multilevel regression.

$\mathrm{N}$ a sociedade contemporânea, caracterizada por intensa disseminação de informações em todos os níveis, as ações de aprendizagem, formais e informais (Coelho Júnior \& Borges-Andrade, 2008a) consolidaram-se como uma das principais ferramentas de aquisição de conhecimentos e habilidades nas organizações. No atual cenário de incertezas, torna-se necessária a criação de ambientes organizacionais que estimulem a aprendizagem entre seus membros e a capacidade de aplicação contínua desta aprendizagem no trabalho.

Assim, a perspectiva de apoio à aprendizagem vai se consolidando, nas organizações, como uma maneira eficiente de se desenvolver competências relacionadas ao trabalho. Deve-se, então, transmitir conhecimentos e habilidades aos indivíduos, a fim de que se incentive constantes processos de inovação e estímulo à aprendizagem. As ações de aprendizagem informal no trabalho passaram a exercer importante efeito nos resultados de trabalho. Nas organizações os indivíduos aprendem o tempo todo, seja informalmente, por imitação, tentativa e erro, conversa com pares e outros atores sociais, ou, mais formalmente, por intermédio de programas planejados de treinamento (Abbad \& Borges-Andrade, 2004).

O desafio, então, consiste em identificar quais variáveis do contexto organizacional otimizam a ocorrência de aprendizagem entre os indivíduos e facilitam novos desempenhos no ambiente de trabalho por meio do apoio organizacional à inovação, experimentação e o desenvolvimento profissional de seus 
integrantes (Morgeson \& Humphrey, 2006). Como toda ação de aprendizagem informal do trabalho deve ser expressa em desempenho (Coelho Júnior \& Borges-Andrade, 2008a), torna-se necessária a modificação constante das práticas organizacionais em torno da contínua adaptação às novas tecnologias. Devese procurar valorizar uma gestão de recursos humanos mais próxima daquele indivíduo que executa seu trabalho, incentivando o seu desenvolvimento contínuo por meio de ações aprendizes, e verificar qual será o impacto destas ações em seu desempenho.

Para a ocorrência da aprendizagem informal no trabalho o suporte à aprendizagem que é provido por colegas e chefias é fundamental (Stacey, 2003). Suporte à aprendizagem diz respeito à percepção do indivíduo quanto ao apoio informal (espontâneo, não planejado) de pares, colegas e chefias à aquisição, retenção, manutenção, generalização e transferência de novos conhecimentos e habilidades às rotinas de trabalho. O quanto o indivíduo percebe de suporte é fundamental ao seu empreendimento em ações aprendizes informais no trabalho.

A literatura na área de comportamento organizacional vem apresentando algumas evidências empíricas acerca das relações preditivas estabelecidas entre variáveis psicológicas e desempenho no trabalho. Nota-se, entretanto, que estas variáveis são comumente investigadas em nível micro de análise, ou seja, são variáveis de nível individual predizendo os resultados de trabalho do indivíduo. Raros são os estudos, como encontrado em Brandão (2008) e Coelho Júnior (2009), que investigam a influência de variáveis de contexto, de nível meso ou macro, na predição de desempenho individual no trabalho.

O presente artigo objetiva testar empiricamente, por meio de modelagem multinível, o efeito de variáveis psicológicas de nível individual e de contexto na predição de desempenho individual no trabalho. Será estudado o tamanho do efeito de variáveis biográficas e profissionais, bem como de percepção de satisfação no trabalho e percepção de suporte à aprendizagem e a interação entre elas na predição de desempenho. Pretende-se verificar o quanto da parcela de variabilidade de desempenho poderá ser explicado por variáveis de nível individual, de contexto e o tamanho do efeito obtido a partir da interação entre variáveis de mesmo nível ou entre níveis (cross-level) analisando-as conjuntamente, como um sistema.

\section{Desempenho no trabalho}

A literatura em desempenho no trabalho aponta que os pesquisadores tem muita dificuldade ao investigarem as causas sistêmicas que podem afetar o desempenho no trabalho, posto que causas individuais são mais comumente encontradas nos relatos da área, segundo Sonnentag e Frese (2002), e Nielsen (2006). Tradicionalmente, os estudos em que a variável "desempenho no trabalho" é investigada como variável critério lançam mão de uma abordagem individual de investigação das variáveis antecedentes (Fonseca, 2001; Macedo, 2007).

Tal fato ocorre provavelmente pelo fato das organizações se preocuparem em aperfeiçoar o desempenho dos indivíduos apenas considerando as suas dificuldades e limitações, não considerando outras variáveis pertencentes a distintos níveis de análise, tais como apontam Yang e Holzer (2006), como o contexto de trabalho e a influência deste sobre o desempenho de um cargo, por exemplo. Analisar somente variáveis de nível individual, na predição de desempenho no trabalho, pode representar uma grande dificuldade para a correta delimitação desta variável, pois credita-se às diferenças individuais algumas questões relacionadas ao comportamento do indivíduo no cargo, mas que podem pertencer a um nível mais macro de análise.

De acordo com DeNisi (2000) o conceito de desempenho é de natureza plural, é multicausal e é constituído por fatores de ordem individual, de contexto e organizacional. Fatores contextuais podem, inclusive, influenciar o modo de agir dos indivíduos e, então, impactar nos seus resultados de trabalho. A partir da análise de Hodgkinson e Healey (2008) depreende-se que um dos objetos de estudo da psicologia no que se refere à investigação de desempenho no trabalho refere-se à análise do compartilhamento de crenças sobre dimensões psicológicas de variáveis antecedentes a desempenho, tais como, no caso deste trabalho, satisfação no trabalho e percepção de suporte à aprendizagem.

A literatura em desempenho individual no trabalho é ainda escassa no que se refere à utilização da abordagem multinível para a investigação de preditores, especialmente quando se relaciona as variáveis "satisfação" e "suporte à aprendizagem informal no trabalho". Nota-se a prevalência da classe de preditores individuais, com foco em características individuais como, por exemplo, raciocínio, características de personalidade, percepção de estilos de liderança e participação em tomadas de decisão, envolvimento com o trabalho e criatividade e comprometimento (Coelho Júnior, 2009).

Satisfação no trabalho vem sendo comumente investigada como preditor de desempenho no trabalho (Brief \& Weiss, 2002; Campbell, Gasser, \& Oswald, 1996; Stajkovic \& Luthans, 2003; Yang \& Holzer, 2006). Quando se analisa a literatura sobre suporte à aprendizagem informal no trabalho, como Ismail (2005) e London e Sessa (2006a), verifica-se que há poucas evidências empíricas relacionando esta variável na testagem de modelos de predição de desempenho no trabalho.

O estado da arte em desempenho humano no trabalho sugere a necessidade de investigação empírica da classe de preditores contextuais afetando os resultados dos indivíduos no trabalho (Nielsen, 2006). Neste trabalho pretende-se contribuir para o estado da arte em relação à variável desempenho medida em nível individual, ao se propor variáveis antecedentes pertencentes a níveis distintos de análise na predição de resultados de trabalho.

\section{Modelagem multinível}

Segundo Hox (2002), as pessoas são influenciadas pelos grupos sociais ou contextos nos quais elas se inserem e que as propriedades desses grupos, por sua vez, são influenciadas pelos indivíduos que os compõem. Como os sistemas podem ser observados em diferentes níveis hierárquicos e as variáveis podem ser definidas isoladamente em cada nível, indivíduos e grupos sociais são tratados conceitualmente como um sistema hierárquico em que os mesmos são particularizados em distintos níveis de análise.

Andrade e Laros (2007) afirmam que, nas análises multiníveis, deve-se especificar a que nível pertencem e quais 
os efeitos diretos das variáveis, bem como quais os efeitos de interação entre os níveis que são esperados. Segundo Hox (2002) torna-se de fundamental valia a correta delimitação do nível do(s) construto(s) de pesquisa a ser investigado. Busca-se identificar, previamente, o nível que cada variável pertence (se individual ou departamental, por exemplo) e o impacto da mesma na predição da variável consequente (critério).

A modelagem multinível considera que variáveis pertencentes a distintos níveis de análise são capazes de explicar a variabilidade de variáveis critérios associadas. Para tal, tornase necessário haver um embasamento teórico que sustente os modelos teóricos multiníveis. Na literatura, verifica-se que há alguns relatos empíricos na área de educação, especialmente em avaliação de programas educacionais, conforme discutido por Andrade e Laros (2007), que vêm utilizando a modelagem multinível na interpretação dos resultados de pesquisa.

O contexto de trabalho, ambiente no qual o indivíduo se encontra inserido e do qual recebe profunda influência, pode ser considerado como um determinante de seu comportamento. Ao adotar modelos multiníveis em pesquisas empíricas, especificase o tamanho do efeito que variáveis dos diferentes níveis têm sobre uma variável critério, no caso deste artigo, desempenho individual no trabalho. A medição das variáveis pode ser feita diretamente no próprio nível da variável e, também, por agregação ou desagregação. Agrega-se uma variável (perspectiva bottom-up) de um nível mais inferior para um nível mais elevado, desagrega-se quando se decompõe a variável de um nível mais alto para um nível mais baixo (perspectiva top-down).

Inicia-se uma análise de dados em uma abordagem multinível por meio do cálculo do modelo vazio, inserindo-se, gradativamente, as variáveis que serão testadas (Hox, 2002). A sequência de inserção de variáveis no modelo inicia-se com variáveis de nível individual, testando sua inclinação fixa e randômica, de nível de contexto e, por fim, testando a interação entre variáveis de mesmo nível ou de níveis distintos. Estes passos são descritos nos resultados desta pesquisa.

A análise multinível é bastante recomendada para estudos voltados à investigação da aprendizagem informal nas organizações, pois parte da pressuposição básica, numa visão interacionista, que o comportamento humano é produto do resultado complexo entre características individuais e de contexto, incluindo-se, nesta última, características objetivas (como o cargo ao qual o indivíduo está lotado, por exemplo) e interpretativas (como a percepção do suporte à aprendizagem informal no trabalho). Neste trabalho serão contempladas hipóteses multiníveis na proposição do modelo teórico que embasou a pesquisa.

\section{Método}

\section{Descrição da organização}

A empresa, pública, de administração indireta, pertence ao ramo de pesquisas agropecuárias. A missão da empresa consiste em viabilizar soluções para o desenvolvimento sustentável do espaço rural, com foco no agronegócio, por meio da geração, adaptação e transferência de conhecimentos e tecnologias, em benefício dos diversos segmentos da sociedade brasileira. A empresa possui, atualmente, 41 unidades descentralizadas espalhadas pelo Brasil, além de três serviços e 11 unidades centrais localizadas na sede.

A empresa conta com um sistema de acompanhamento e avaliação de desempenho pautado nas etapas de planejamento, execução, avaliação e revisão de desempenho. A medida de desempenho ou variável critério deste estudo refere-se ao índice de resultados alcançados (IRA), que se refere à avaliação individual com foco no cumprimento do plano de trabalho.

\section{Procedimentos de coleta de dados}

A coleta de dados, realizada totalmente via e-mail, em virtude de sua maior abrangência e alcance, foi feita por meio da estratificação da amostra de funcionários da Empresa utilizando como critério o grau de escolaridade. Funcionários com ensino fundamental, cuja maioria não tinha $e$-mails, foram eliminados do banco de dados da pesquisa. A pesquisa foi realizada no próprio ambiente virtual da empresa.

A escala de suporte à aprendizagem (Coelho Júnior \& Borges-Andrade, 2008b) e de satisfação no trabalho (Siqueira, 1985) foram digitalizados no ambiente virtual da empresa. A escala de suporte à aprendizagem continha 31 itens, com dois fatores (suporte à aprendizagem da chefia, com 25 itens e alpha de 0,98, e suporte dos colegas, com 6 itens e alpha de $0,97)$ e cargas fatoriais oscilando entre 0,59 a 0,98 . A escala de satisfação no trabalho continha 25 itens divididos em cinco fatores: satisfação quanto ao relacionamento interpessoal ( 5 itens, alph $a=0,89)$, quanto à política de remunerações ( 5 itens, $a l p h a=0,95)$, quanto à chefia $(5$ itens, alpha $=0,95)$, quanto à natureza da tarefa (4 itens, alpha $=0,85)$ e quanto à política de promoções (5 itens, alpha $=0,92$ ).

Quando o indivíduo recebia o e-mail da pesquisa e clicava no link de acesso aos questionários, era encaminhado à primeira tela da pesquisa, que solicitava a digitação de seu nome de usuário de rede da empresa e número de matrícula funcional. $\mathrm{O}$ número de matrícula foi o vínculo utilizado para parear as respostas dos questionários com a nota bruta de desempenho que foi fornecida pela organização. O sigilo das respostas foi plenamente garantido e reforçado continuamente.

\section{Caracterização da amostra}

A maioria dos participantes eram homens $(58,9 \%)$, com escolaridade mínima de nível superior $(54,6 \%)$ e idade média de 45,8 anos $(D P=9,15)$. A maioria possuía acima de 16 anos de trabalho na empresa (64,2\%), sendo o tempo médio de 17,2 anos $(D P=10,11)$. A maior parte eram Assistentes $C$ seguidos pelos Assistentes A e Pesquisadores A. Obtiveram-se respostas válidas oriundas de 808 participantes distribuídos em 45 unidades centralizadas e descentralizadas da empresa. Verificou-se grande variabilidade no perfil amostral, com participantes lotados em unidades da empresa distribuídos por todo o Brasil.

\section{Procedimentos de análise de dados}

Após a realização de análises descritivas e exploratórias procedeu-se o cálculo dos escores fatoriais, com o objetivo de reduzir o número de variáveis que seriam testadas nos modelos 
empíricos. Para a análise multinível procedeu-se à execução dos seis passos sugeridos por Hox (2002) e Andrade e Laros (2007). Agregaram-se variáveis biográficas, profissionais e de suporte à aprendizagem. Foram identificados e analisados o impacto dos casos extremos uni e multivariados nos resultados, optando-se pela apresentação dos mesmos sem a presença de tais casos extremos.

\section{Resultados}

Procedeu-se ao cálculo dos escores fatoriais para cada um dos fatores obtidos para as escalas de suporte à aprendizagem e satisfação no trabalho. Variáveis pessoais e profissionais, categóricas, foram recodificadas como dummies, de acordo com a Tabela a seguir.

Tabela 1

Recodificação de variáveis pessoais e profissionais.

\begin{tabular}{ll}
\multicolumn{1}{c}{ Descrição das variáveis } & \multicolumn{1}{c}{ Recodificação } \\
\hline Variáveis do nível individual & Variáveis recodificadas \\
& $0=$ masculino; 1 = feminino \\
Grau de Escolaridade REC 1 & $0=$ superior, superior com \\
& $\begin{array}{l}\text { especialização, mestrado, } \\
\text { doutorado e pós-doutorado } \\
\text { igual a zero } \\
1=\text { ensino médio }\end{array}$ \\
& \\
Grau de Escolaridade REC 2 & $0=$ mestrado, doutorado e \\
& pós-doutorado \\
$1=$ ensino médio, superior e & superior com especialização
\end{tabular}

Fator 1 Suporte à Aprendizagem desvio indivíduo (Suporte das Chefias e Unidade de Trabalho)

Fator 2 Suporte à Aprendizagem desvio indivíduo (Suporte dos Colegas)

Cargo REC 1
Resultado da diferença entre percepções coletivas de suporte à aprendizagem das chefias e Unidade e percepções individuais

Resultado da diferença entre percepções coletivas de suporte à aprendizagem dos colegas e percepções individuais

$0=$ pesquisador $\mathrm{A} \mathrm{e}$ pesquisador $\mathrm{B}$; $1=$ analista $\mathrm{A}$, analista $\mathrm{B}$, assistente $\mathrm{A}$, assistente $\mathrm{B}$, assistente $\mathrm{C}$
Os dados sinalizam que o desempenho médio relativo ao índice de resultados alcançados pelos indivíduos é de 81,969. A estimativa de variância entre as unidades é 179,125 e da variância entre os funcionários é 364,487. O modelo vazio está representado na Tabela 2 . Os dados referentes ao intercepto podem ser interpretados como o valor esperado do desempenho do indivíduo quanto aos resultados alcançados.

Considerando-se estes dados pode-se, então, proceder ao cálculo do coeficiente de correlação intraclasse (i.c.c.). Este coeficiente indica o quanto da variação total nos escores atribuídos aos resultados alcançados se deve a diferenças entre as unidades pesquisadas. Este coeficiente é obtido por:

De acordo com os dados encontrados $33 \%$ da variação total dos escores relativos ao IRA são atribuídos em função da unidade de trabalho. $\mathrm{O}$ fato de estar lotado em determinada unidade da empresa exerce efeito significativo quanto ao desempenho alcançado pelo indivíduo. A estimativa de $-2 *$ Loglikelihood (deviance) para o modelo vazio foi de 7053.077.

Após o cálculo do modelo vazio procedeu-se à inserção de variáveis de controle e variáveis explicativas de nível individual, com efeito fixo. Os resultados podem ser visualizados na Tabela 3.

$$
\text { i.c.c. }=\frac{\sigma^{2} u 0}{\sigma^{2} u 0+\sigma^{2} \mathrm{e}}=\frac{179,125}{179,125+364,487}=0,33
$$


Tabela 2

Estimativas para o Modelo Vazio (Variável Critério IRA)

\begin{tabular}{cccc}
\hline Efeito fixo & Coeficiente $(\beta)$ & $D P$ & \\
\hline Y00 = Intercepto & 81,969 & 2,127 & Razão $t$ \\
$\begin{array}{c}\text { Efeito Randômico } \\
\text { Variância do nível 2: } \\
\sigma^{2} \mathrm{u}_{0=\text { variância (U0j) }}\end{array}$ & Variância & $D P$ & 4,19 \\
$\begin{array}{c}\text { Variância do nível 1: } \\
\sigma_{\mathrm{e}=\text { variância (Rij) }}^{2}\end{array}$ & 179,125 & 42,728 & 19,4 \\
& 364,487 & 18,810 & \\
\hline $\begin{array}{l}\text { Deviance (- 2log } \\
\text { verossimilhança) }\end{array}$ & & & \\
\hline
\end{tabular}

Tabela 3

Comparação entre os Modelos Vazio, Modelo 2 e Modelo 3 (Variável Critério IRA)

\begin{tabular}{|c|c|c|c|c|c|c|}
\hline \multirow{2}{*}{$\begin{array}{l}\text { Variáveis Explicativas } \\
\text { Parâmetro Fixo }\end{array}$} & \multicolumn{2}{|c|}{ Modelo 1 (Vazio) } & \multicolumn{2}{|c|}{$\begin{array}{l}\text { Modelo } 2 \text { (Referência): } \\
\text { Adicionando Variáveis de } \\
\text { Controle }\end{array}$} & \multicolumn{2}{|c|}{$\begin{array}{c}\text { Modelo } 3 \text { (Condicional) } \\
\text { Adicionando Variáveis } \\
\text { Explicativas do Nível } 1\end{array}$} \\
\hline & $\begin{array}{c}\text { Efeito }(\beta) \text { e Erro } \\
\text { Padrão }\end{array}$ & Razão $t$ & $\begin{array}{c}\text { Efeito }(\beta) \text { e Erro } \\
\text { Padrão }\end{array}$ & Razão $t$ & $\begin{array}{l}\text { Efeito }(\beta) \text { e Erro } \\
\text { Padrão }\end{array}$ & Razão $t$ \\
\hline \multirow{2}{*}{$\begin{array}{l}\text { Intercepto } \\
\text { Idade }\end{array}$} & $81,9(2,1)$ & --- & $73,3(2,2)$ & ---- & - & - \\
\hline & & & $-1,1(0,7)$ & $-1,61$ & - & - \\
\hline Gênero & & & $0,7(1,4)$ & 0,49 & - & - \\
\hline CargoREC1 (Controle) & & & $7,9(2,5)$ & $3,18 *$ & - & - \\
\hline $\begin{array}{l}\text { GrauEscol.REC1 } \\
\text { (Controle) }\end{array}$ & & & $-2,7(1,9)$ & $-1,36$ & - & - \\
\hline $\begin{array}{l}\text { GrauEscol.REC2 } \\
\text { (Controle) }\end{array}$ & & & $6,5(2,4)$ & $2,70^{*}$ & - & - \\
\hline Tempo de serviço & & & & & $-0,55(0,66)$ & $-0,83$ \\
\hline Satisfação (Fator 1) & & & & & $0,80(0,66)$ & 1,22 \\
\hline Satisfação (Fator 2) & & & & & $-0,67(0,67)$ & $-0,99$ \\
\hline Satisfação (Fator 3) & & & & & $-0,28(0,66)$ & $-0,43$ \\
\hline Satisfação (Fator 4) & & & & & $0,14(0,66)$ & 0,21 \\
\hline Satisfação (Fator 5) & & & & & $-0,57(0,66)$ & 0,87 \\
\hline $\begin{array}{l}\text { Suporte das Chefias } \\
\text { (Fator1) }\end{array}$ & & & & & $-0,27(0,63)$ & $-0,42$ \\
\hline $\begin{array}{l}\text { Suporte dos Colegas } \\
\text { (Fator2) }\end{array}$ & & & & & $-0,85(0,63)$ & $-1,34$ \\
\hline Parâmetro Aleatório & Variância e Erro Padrão & Razão $t$ & $\begin{array}{l}\text { Variância e Erro } \\
\text { Padrão }\end{array}$ & Razão $t$ & $\begin{array}{l}\text { Variância e Erro } \\
\text { Padrão }\end{array}$ & Razão $t$ \\
\hline Variância do nível 2 & $179,1(42,7)$ & $4,19 *$ & $174,1(41,2)$ & $4,22 *$ & - & - \\
\hline Variância do nível 1 & $364,5(18,8)$ & $19,38^{*}$ & $321,8(16,6)$ & $19,38^{*}$ & - & - \\
\hline \multicolumn{7}{|l|}{ Ajuste do Modelo } \\
\hline Deviance & 7053.077 & & 6957 & & - & \\
\hline $\begin{array}{l}\text { Número de parâmetros } \\
\text { estimados }\end{array}$ & 3 & & & & - & \\
\hline Diferença entre deviances & & & & & - & \\
\hline $\begin{array}{l}\text { Diferença entre } \mathrm{n}^{\circ} \mathrm{de} \\
\text { parâmetros (g.1.) }\end{array}$ & & & & & - & \\
\hline Teste $x^{2}$ & & & & $6^{*}$ & - & \\
\hline
\end{tabular}


Comparando-se os resultados entre o modelo vazio e o modelo referência verifica-se uma redução bastante significativa da deviance (de 7053.077 para 6957.855), o que indica um melhor ajuste do modelo referência em relação ao modelo vazio. O tipo de cargo ao qual o indivíduo se encontra lotado apresentou contribuição positiva $(7,898)$ relativo ao índice de resultados alcançados. Os Analistas A e B, bem como os Assistentes A, B e $\mathrm{C}$ apresentaram maiores escores 'IRA' quando comparados aos Pesquisadores A e B. O grau de escolaridade $(6,462)$ também contribuiu significativamente para a variância do IRA. Indivíduos com ensino médio, superior completo e superior com especialização obtiveram maiores escores IRA de desempenho em relação àqueles com mestrado, doutorado e pós-doutorado.

Todas as variáveis de nível individual não apresentaram contribuição significativa $(t>2)$ na predição de variância da variável critério IRA. Assim, o Modelo 2 será utilizado como referência em comparação aos modelos seguintes. Conforme recomenda Hox (2002) foram inseridas variáveis de nível 2

Tabela 4

Comparação entre o Modelo 4, Modelo 5 e Modelo Final (Variável Critério IRA)

\begin{tabular}{|c|c|c|c|c|c|c|}
\hline \multirow{2}{*}{$\begin{array}{l}\text { Variáveis Explicativas } \\
\text { Parâmetro Fixo }\end{array}$} & \multicolumn{2}{|c|}{$\begin{array}{c}\text { Modelo 4: adicionando } \\
\text { variáveis explicativas do nível } \\
2\end{array}$} & \multicolumn{2}{|c|}{$\begin{array}{l}\text { Modelo 5: efeito } \\
\text { randômico variáveis } \\
\text { nível } 1\end{array}$} & \multicolumn{2}{|c|}{$\begin{array}{l}\text { Modelo final - efeito de } \\
\text { interação }\end{array}$} \\
\hline & $\begin{array}{l}\text { Efeito }(\beta) \text { e Erro } \\
\text { Padrão }\end{array}$ & Razão $t$ & $\begin{array}{l}\text { Efeito }(\beta) \text { e } \\
\text { Erro } \\
\text { Padrão }\end{array}$ & Razão $t$ & $\begin{array}{l}\text { Efeito }(\beta) \\
\text { e Erro } \\
\text { Padrão }\end{array}$ & Razão $t$ \\
\hline Intercepto & $66,9(3,6)$ & ---- & - & - & $67,7(3,6)$ & ---- \\
\hline CargoREC1 (Controle) & $7,8(2,5)$ & $3,16^{*}$ & - & - & $6,9(2,4)$ & $2,9^{*}$ \\
\hline GrauEscREC1 (Controle) & $-2,7(1,9)$ & $-1,36$ & - & - & $4,1(1,9)$ & $2,1^{*}$ \\
\hline GrauEscREC2 (Controle) & $6,3(2,4)$ & $2,65^{*}$ & - & - & $7,9(2,4)$ & $3,3^{*}$ \\
\hline GrauEscREC1_agregado & $48,4(21,9)$ & $2,2 *$ & - & - & $47,1(21,9)$ & $2,1^{*}$ \\
\hline Fator 2 Suporte_agregado & $3,5(1,7)$ & $2,0^{*}$ & - & - & $7,8(1,9)$ & $4,1^{*}$ \\
\hline GrauEscREC2\&Fator2Suporte_agregado & - & - & - & - & $-7,0(1,3)$ & $-5,2 *$ \\
\hline $\begin{array}{l}\text { Variância - intercepto } \\
\text { Variância do nível } 1\left(\mathrm{R}_{\mathrm{ij}}\right)\end{array}$ & $\begin{array}{l}142,8(34,6) \\
321,9(16,6)\end{array}$ & $\begin{array}{r}4,1^{*} \\
19,4^{*}\end{array}$ & $\begin{array}{l}- \\
-\end{array}$ & - & $\begin{array}{l}141,0(34,0) \\
309,2(15,9)\end{array}$ & $\begin{array}{r}4,1^{*} \\
19,4^{*}\end{array}$ \\
\hline \multicolumn{7}{|l|}{ Ajuste do Modelo } \\
\hline Deviance & 6950.299 & & - & & - & 6919.250 \\
\hline Número de parâmetros estimados & & & - & & - & 9 \\
\hline Diferença entre deviances & 7.556 & & - & & - & 31.049 \\
\hline Diferença entre $\mathrm{n}^{\circ}$ de parâmetros (g.l.) & 2 & & - & & - & 2 \\
\hline Teste $x^{2}$ & 3,8 & & - & & - & $15,5^{*}$ \\
\hline
\end{tabular}

(passo 4), posteriormente variáveis aleatórias ou randômicas (passo 5) e, por fim, interações entre variáveis pertencentes ao mesmo nível de análise e variáveis cross-level. Os resultados estão apresentados na Tabela 4.

Comparando-se o Modelo 2 com o Modelo 4 verifica-se uma redução bastante significativa da deviance (de 6957.855 para 6950.299), o que é um importante indicador numérico acerca do melhor ajuste do modelo ao se inserir variáveis explicativas de nível $2\left(\chi_{2}^{2}-\chi_{4}^{2}\right.$ / g.l $\left.=3,8\right)$. As variáveis de controle que contribuíram significativamente no Modelo 2 mantiveram-se significativas no Modelo 4.

Com relação às percepções coletivas de suporte, ou seja, relativas ao nível 2 (unidades de trabalho), as mesmas exerceram um efeito significativo direto $(3,484)$ sobre a variável critério IRA. Os dados sinalizam que as unidades nas quais os indivíduos compartilham percepções favoráveis quanto à ocorrência de condições de apoio à aprendizagem informal foram caracterizadas pelos melhores escores de desempenho. $\mathrm{O}$ ambiente de trabalho apresentou forte relação com o índice de resultados alcançados pelos indivíduos em situação de trabalho.

O grau de escolaridade exerceu influência no desempenho quando considerado como variável de contexto. O efeito positivo exercido por esta variável no nível $2(48,404)$ indica que há variabilidade entre as unidades investigadas referentes ao impacto da escolarização na nota de desempenho. Em unidades caracterizadas por ter funcionários com menor grau de escolarização (funcionários com ensino médio e superior) obteve-se os maiores escores dos indivíduos no que se refere aos resultados alcançados.

Todas as variáveis de nível 2 que não apresentaram contribuição significativa $(t>2)$ na predição de variância da variável critério IRA foram estimadas novamente. Nota-se uma redução bastante significativa da variância do nível 2 comparando-se o Modelo 2 em relação ao Modelo 4, o que indica haver um melhor ajuste deste modelo optando-se pelo mesmo para comparações subsequentes de ajustamentos de modelos.

Após a inserção de variáveis randômicas (passo 5, segundo Hox, 2002) verificou-se que nenhuma variável de nível 1 com efeito aleatório foi significativa $(p<0,05)$. Optou-se pelo descarte do Modelo 5, mantendo-se a estrutura empírica encontrada no Modelo 4. As variáveis randômicas serão estimadas novamente em modelos subseqüentes. 
O Modelo final relativo ao escore de desempenho IRA apresentou um maior número de parâmetros estimados em relação ao Modelo 4, assim como houve uma redução bastante significativa da deviance (de 6950.299 para 6919.250), do ajuste do modelo $\left(\chi_{4}{ }_{4}-\chi_{6 / \text { g.l }}=15,5\right)$ e das variâncias de nível 2 e de nível 1. Dos efeitos de interação testados no Modelo final (Modelo 6), apenas o efeito entre grau de escolaridade recodificada 2 e percepções coletivas de suporte à aprendizagem provido por colegas $(-7,020, t=-5,2)$ foi significativo. Todas as outras interações abrangendo variáveis do mesmo nível e variáveis cross-level que não foram significativas $(t<2)$ foram descartadas.

A interação negativa sinaliza que o efeito obtido no desempenho em IRA em virtude do grau de escolaridade (mestrado, doutorado e pós-doutorado) é menor quando há a percepção positiva compartilhada entre os indivíduos sobre o suporte à aprendizagem entre colegas. Os mais bem avaliados em IRA estão lotados em unidades caracterizadas por terem o compartilhamento do suporte à aprendizagem entre os indivíduos e este compartilhamento tem maior efeito quando é menor a escolarização entre seus funcionários. Tal resultado indica que não apenas o fato de pertencer a um ambiente em que há percepções coletivas favoráveis ao provimento de suporte à aprendizagem ou se ter menor escolarização são capazes de impactar na obtenção de melhores escores em IRA, mas sim que as percepções compartilhadas de suporte em nível de contexto devem ser analisadas conjuntamente com o grau de escolaridade dos indivíduos. O tamanho dos efeitos destas variáveis medidas separadamente não exerce o mesmo impacto do que quando se considera tais variáveis interagindo e compartilhando variância em IRA. Em estudos futuros a interação entre estas variáveis deve ser tratada como uma única variável, não mais isoladamente.

Pode-se perceber, analisando o Modelo final, que, uma vez controladas as variáveis que representam características profissionais, as percepções coletivas referentes a suporte à aprendizagem dos colegas tornaram-se significativas com uma contribuição positiva $(7,799)$. Ou seja, o efeito da percepção compartilhada de suporte à aprendizagem em IRA ocorre em virtude de características específicas de cada Unidade.

Porém, é interessante verificar que a contribuição da variável agregada de suporte à aprendizagem é positiva quando a mesma é inserida isoladamente no modelo, diferentemente de quando a mesma foi inserida em interação com o grau de escolaridade dos indivíduos, quando sua contribuição se tornou negativa. Hipotetiza-se que suporte à aprendizagem analisado isoladamente, enquanto característica de contexto, pode ser considerado como um importante preditor de variância em IRA. Todavia, quando em interação com o grau de escolaridade dos indivíduos, seu efeito é minimizado, posto que os dados indicam que o nível de escolarização do indivíduo parece ser determinante de seu escore de desempenho relativo ao índice de resultados alcançados no trabalho.

Essa afirmação pode ser corroborada quando se observam mais detalhadamente os dados obtidos no Modelo final. Os dados sinalizam que após a inserção do termo de interação a variável grau de escolaridade recodificada 1 também passou a contribuir significativamente para a predição de variância relativa ao IRA
$(4,109)$. A variável agregada grau de escolaridade recodificada 1 também contribuiu significativamente $(47,113)$ no Modelo final, indicando que em unidades da empresa em que há maior contingente de pessoas com ensino médio pode haver um modus operandi específico que diferencia o desempenho dos indivíduos em IRA quando comparado com outras unidades da empresa em que não há tal influência no contexto a partir do grau de escolaridade do indivíduo.

Outra possibilidade refere-se a que o compartilhamento das crenças sobre o suporte à aprendizagem não é suficiente para impactar positivamente no desempenho. A escolaridade parece determinar o impacto das crenças sobre a nota IRA e parece indicar a existência de duas subculturas organizacionais, uma representada pelos pesquisadores, com mestrado, doutorado e pós-doutorado, e outra particularizada no restante dos funcionários. Recomenda-se a realização de estudos posteriores que considerem o tamanho do efeito em IRA de cada um dos níveis de escolaridade separadamente, não de maneira conjunta tal como realizado neste trabalho.

Em síntese, o compartilhamento de variância entre variáveis de nível 1 e 2 sinaliza que fatores de contexto, quando analisados conjuntamente a fatores individuais, são capazes de explicar importante variância do desempenho relativo aos resultados alcançados pelo indivíduo. Há um padrão cultural possivelmente determinado pelo tempo de trabalho na empresa que é capaz de naturalizar os comportamentos socialmente aceitáveis no âmbito da empresa. Variáveis individuais ou de contexto, separadamente ou em interação, são capazes de produzir efeito significativo na predição de variância relativa ao IRA.

\section{Discussão}

Os resultados sinalizaram que funcionários com ensino médio, superior e superior com especialização obtiveram maiores notas em relação aos demais. Resultados semelhantes em que o grau de escolaridade e anos de estudo predisseram desempenho foram encontrados por Fonseca (2001) e Macedo (2007). Em estudos posteriores sugere-se que cada categoria de escolaridade seja analisada separadamente, com o objetivo de investigar o impacto de cada uma delas na predição de desempenho na Empresa. Hipotetiza-se que a recodificação das variáveis realizadas neste estudo pode ter dificultado a interpretação dos resultados. A investigação individual do grau de escolaridade sem qualquer tipo de recodificação se faz necessária, a fim de verificar o tamanho do efeito de cada uma delas. Parece haver um efeito do grau de escolaridade no escore final de IRA, mas, em virtude da recodificação realizada, tal conclusão não pôde ser realizada.

As maiores notas de desempenho foram atribuídas aos Analistas A e B e aos Assistentes A, B e C (área meio). Uma das explicações para esta diferença na atribuição de escores entre cargos meio e fim pode estar nas meta-análises (ver Sonnentag \& Frese, 2002), que indicam que há uma relação preditiva positiva entre características e finalidade do trabalho (como o desenho de tarefas, por exemplo) e desempenho. Em outras meta-análises apresentam-se correlações positivas entre intervenções no contexto organizacional (como o redesenho contínuo das tarefas 
de um cargo entre chefia e subordinados, segundo Morgeson \& Humphrey, 2006) e seu impacto no desempenho. Porém, nas meta-análises não se pode estabelecer uma interpretação causal, pois tratam-se de estudos correlacionais.

Outras pesquisas como Macedo (2007) e Nielsen (2006) também investigaram o efeito do tipo do cargo sobre a avaliação de desempenho. Estes achados sugerem que variáveis relativas ao cargo (por exemplo, tipo de cargo, grau de ambiguidade das tarefas, autonomia decisória, controle da tarefa, significado do trabalho) são melhores preditoras de desempenho quando comparadas a outras variáveis pessoais ou profissionais.

Estudos tanto nacionais quanto internacionais (Hodgkinson \& Healey, 2008; Ismail, 2005; Macedo, 2007; Sonnentag \& Frese, 2002) ressaltam a importância de se prover suporte às ações informais de aprendizagem a fim de que haja efetivamente impacto no desempenho da tarefa. A relação teórica entre o ambiente de aprendizagem e desempenho foi proposta por Sonnentag e Freese (2002) e confirmada empiricamente no âmbito desta pesquisa. A percepção de suporte à aprendizagem, até então investigada como sendo uma variável de nível individual, passa a ser considerada como uma construção social coletiva e compartilhada pelos indivíduos nas unidades, que sistematicamente impacta no desempenho dos mesmos. $\mathrm{O}$ fato dos indivíduos estarem num mesmo grupo social de trabalho faz com que estejam submetidos a estímulos semelhantes, logo compartilhem percepções. Estes estímulos exercem influência sobre os padrões de comportamento, de modo que a atuação de cada indivíduo deve ser analisada a partir do efeito das características do contexto.

O clima de confiança e respeito mútuo, segundo Stacey (2003) e London e Sessa (2006a), deve ser continuamente reforçado. As relações de amizade e confiança entre pares e colegas buscam reforçar comportamentos de apoio ou de incentivo a qualquer ação que esteja relacionada à aprendizagem no trabalho.

Outra variável de nível de contexto que também exerceu efeito significativo no escore de desempenho IRA correspondeu ao grau de escolaridade. Este achado sugere que unidades que têm mais pessoas com menor grau de escolaridade poderiam estar provendo mais pessoal de apoio e que tal apoio resultaria em melhores indicadores de desempenho para todos. Encontrou-se interação significativa entre grau de escolaridade e percepções coletivas de suporte à aprendizagem provido pelos colegas. A contribuição desta interação se deu de forma negativa, sugerindo que o tamanho do efeito sobre o desempenho de funcionários com mestrado, doutorado e pós-doutorado é menor quando existe um maior compartilhamento de crenças sobre suporte à aprendizagem entre colegas de lotação. Hipotetizase que pessoas com pós-graduação stricto sensu tendem a ser mais independentes quanto à execução de suas tarefas. Este resultado é importante, pois parece indicar que os contextos organizacionais de provimento de suporte à aprendizagem têm efeitos especialmente positivos sobre o desempenho de pessoas que não têm pós-graduação estrito senso.

O presente estudo também testou o efeito randômico das variáveis psicológicas suporte à aprendizagem e satisfação no trabalho, bem como das variáveis biográficas e funcionais.
Não foi encontrada nenhuma contribuição estatisticamente significativa.

Sugere-se, em estudos posteriores, que sejam acrescentadas novas variáveis que, possivelmente, possam ter efeito significativo na predição de desempenho quando analisadas em interação. Recomenda-se que sejam incorporadas as estratégias informais de aprendizagem 'busca de ajuda interpessoal' e 'busca de ajuda em material escrito', e que as mesmas sejam propostas como termo de interação com as variáveis "grau de escolaridade" e "percepções compartilhadas de suporte" tanto dos colegas quanto das chefias na predição de desempenho.

\section{Conclusões}

Este trabalho estabeleceu como objetivo investigar as relações entre variáveis relativas ao indivíduo e contexto na predição de desempenho individual. Investigou-se o efeito da satisfação no trabalho e do suporte à aprendizagem, medidas no nível individual, na predição de desempenho no trabalho, assim como de variáveis biográficas, funcionais e das percepções coletivas de suporte à aprendizagem.

Pode-se afirmar, analisando o modelo multinível final, que a relação teórica hipotetizada entre suporte à aprendizagem e desempenho foi confirmada empiricamente. As percepções coletivas de suporte informal à aprendizagem, enquanto variável de contexto, foram capazes de predizer desempenho. Este é o achado mais consistente e importante deste artigo.

Foram identificados os tamanhos dos efeitos associados a cada uma das variáveis psicológicas que contribuíram significativamente nos modelos multiníveis. Comparando às demais, a variável psicológica "percepções coletivas de suporte dos colegas à aprendizagem”, de nível de contexto, foi a que mais contribuiu significativamente para predição de desempenho individual no trabalho. Tal resultado indica que estas percepções compartilhadas estão fortemente associadas a desempenho. Isto pode ter sido potencializado em virtude do controle das variáveis cargo e grau de escolaridade.

O escore IRA é influenciado principalmente pelo grau de escolaridade. Quatro das variáveis que predisseram este escore são relativas a grau de escolaridade, isoladamente ou em interação. Possivelmente esta característica exerce este efeito porque o IRA, que se refere à avaliação individual com foco no cumprimento do plano de trabalho por meio de critérios de eficiência e eficácia, seria dependente do perfil de competências necessárias ao ocupante do cargo. Este seria determinado pelo nível de instrução dos indivíduos, que é pré-requisito para ocupação dos cargos.

A importância deste trabalho justificou-se na necessidade de investigação dos principais preditores de desempenho no trabalho contemplando-se, no desenho de pesquisa, variáveis pertencentes aos níveis menos elevados (individual) e mais elevados (unidades de trabalho). Espera-se que haja implicações teóricas neste trabalho, problematizando-se a importância da análise de variáveis de contexto (especialmente suporte à aprendizagem) na predição de desempenho no trabalho.

Há importantes implicações práticas a serem consideradas. A primeira delas refere-se ao papel dos gestores de recursos 
humanos da organização no que tange às ações de apoio à aprendizagem informal do trabalho. Tais ações devem ser continuamente reforçadas pelos gestores da organização, visto que este trabalho apontou a importância da criação de um ambiente de aprendizagem que estimule o suporte psicossocial dos colegas.

Outra implicação prática diz respeito à necessidade da empresa adotar políticas de gestão do capital intelectual visando estimular o amplo compartilhamento informal e disseminação do conhecimento. A apropriação e internalização do conhecimento, bem como o aprendizado em grupo, devem ser estimulados especialmente em unidades cujas percepções coletivas de suporte não se configuraram como prática compartilhada. Espera-se que estes resultados sejam reconhecidos não apenas pelos gestores da empresa investigada mas, também, por outros gestores organizacionais interessados nas relações entre variáveis individuais, de contexto e seu impacto no desempenho.

Como contribuições, o presente estudo demonstrou empiricamente a importância que variáveis psicológicas provenientes de diferentes níveis exercem na explicação do desempenho individual, o que pode ser considerado como avanço nas pesquisas em comportamento organizacional. Os resultados dos coeficientes de correlação intraclasse (i.c.c.) sugerem, porém, que outras variáveis de nível de contexto que não foram estudadas nesta tese podem ser capazes de predizer parcela significativa de desempenho na Empresa.

Como limitações, verificou-se que a decisão metodológica de solicitar o número da matrícula do indivíduo na coleta de dados pode ter feito com que vários respondentes tenham desistido de participar, com receio de sofrerem algum tipo de retaliação. Acredita-se que a medida de satisfação no trabalho pode ter potencializado este receio por parte dos indivíduos e contribuído substancialmente para a não resposta completa aos instrumentos.

Outra limitação identificada refere-se à falta de informações referentes à composição dos grupos de respondentes por unidade investigada e aos padrões de interação social estabelecidos entre eles (por exemplo, nível de coesão grupal e grau de interação social entre os membros das unidades, dentre outros). Pode ser que a incorporação de outras variáveis referentes à composição de cada grupo fornecesse resultados mais abrangentes considerando cada realidade organizacional, obtendo-se indícios de formação de identidade social ou de outros tipos de crenças compartilhadas (por exemplo, crenças sobre a efetividade do grupo) que poderiam agregar valor nas análises realizadas. Recomenda-se que as mesmas sejam posteriormente testadas em modelos multiníveis de predição de desempenho.

\section{Referências}

Abbad, G., \& Borges-Andrade, J. E. (2004). Aprendizagem humana nas organizações e trabalho. In: J. C. Zanelli, J. E. Borges-Andrade, \& A. V. B. Bastos (Orgs), Psicologia, Organizações e Trabalho no Brasil (pp.237-275). Porto Alegre: Artmed.

Andrade, J. M., \& Laros, J. A. (2007). Fatores associados ao desempenho escolar: estudo multinível com dados do SAEB/2001. Psicologia: Teoria e Pesquisa,
23(1), 33-42.

Brandão, H. P. (2008). Aprendizagem, contexto, competência e desempenho: um estudo multinível. (Tese de Doutorado não publicada). Universidade de Brasília, Brasília.

Brief, A. P., \& Weiss, H. M. (2002). Organizational Behavior: affect at work. Annual Review of Psychology, 53, 279-307.

Campbell, J. P., Gasser, M. B., \& Oswald, F. L. (1996). The substantive nature of job performance variability. In: K. R. Murphy (Org.), Individual Differences and Behavior in Organizations (pp.258-299). San Francisco: Jossey-Bass.

Coelho Júnior, F. A., \& Borges-Andrade, J. E. (2008a). Uso do conceito de aprendizagem em estudos organizacionais. Paidéia, 40(18), 221-234.

Coelho Júnior, F. A., \& Borges-Andrade, J. E. (2008b). Re-validação de escala de suporte à aprendizagem informal no trabalho. Trabalho apresentado no XXXII Encontro Nacional de Programas de Pós-Graduação em Administração (Enanpad), Rio de Janeiro.

Coelho Júnior, F. A. (2009). Suporte à aprendizagem, satisfação no trabalho e desempenho: um estudo multinível (Tese de doutorado não publicada). Universidade de Brasília, Brasília.

DeNisi, A. S. (2000). Performance appraisal and performance management: a multilevel analysis. In K. J. Klein \& S. W. J. Kozlowski (Orgs.), Multilevel theory, research and methods in organizations: foundations, extensions and new directions (pp. 121-156). San Francisco: Jossey-Bass.

Fonseca, C. A. M. (2001). Criatividade e comprometimento organizacional: suas relações com a percepção de desempenho no trabalho (Dissertação de Mestrado não publicada). Universidade Federal da Bahia, Salvador.

Hodgkinson, G. P., \& Healey, M. P. (2008). Cognition in organizations. Annual Review of Psychology, 59, 387-417.

Hox, J. (2002). Multilevel Analysis:techniques and applications. Lawrence Erlbaum Associates: New Jersey.

Ismail, M. (2005). Creative climate and learning organization factors: their contribution toward innovation. Leadership \& Organization Development Journal, 26(8), 639-654.

London, M., \& Sessa, V. I. (2006). Continuous learning in organizations: a living systems analysis of individual, group and organization learning. Research in Multi-Level Issues, 5, 123-172.

Macedo, R. B. (2007). Modelo de avaliação de impacto da aprendizagem no nível de resultados organizacionais: preditores relacionados ao indivíduo e ao contexto organizacional (Tese de Doutorado não publicada). Universidade de Brasília, Brasília.

Morgeson, F. P., \& Humphrey, S. E. (2006). The work design questionnaire (WDQ): developing and validating a comprehensive measure for assessing job design and the nature of work. Journal of Applied Psychology, 91, 1321-1339.

Nielsen, S. K. (2006). A Multisource model of perceived organizational support and performance (Dissertação de mestrado não publicada). Universidade do Tennessee, Knoxville.

Siqueira, M. M. M. (1985). Antecedentes de comportamento de cidadania organizacional: a análise de um modelo pós-cognitivo (Tese de doutorado não publicada). Universidade de Brasília, Brasília.

Sonnentag, S., \& Frese, M. (2002). Performance concepts and performance theory. In: S. Sonnentag (Org.), Psychological management of individual performance (pp.3-27). Great Britain: John Wiley \& Sons Ltda.

Stacey, R. (2003). Learning as an activity of interdependent people. The Learning Organization, 10(6), 325-331.

Stajkovic, A. D., \& Luthans, F. (2003). Behavioral management and task performance in organizations: conceptual background, meta-analysis, and test of alternative models. Personnel Psychology, 56, 155-194.

Yang, K., \& Holzer, M. (2006). The performance-trust link: implications for performance measurement. Public Administration Review, 66(1), 114-126. 
Francisco Antonio Coelho Júnior, doutor em Psicologia Social, do Trabalho e Organizacional pela Universidade de Brasília, é professor Adjunto na mesma universidade. Endereço para correspondência: Universidade de Brasília, Campus Universitário Darcy Ribeiro. Instituto Central de Ciências (Ala Norte), Departamento de Administração, Programa de Pós-Graduação em Administração. CEP: 70910-900. BrasíliaDF. Tels.: (61) 3107-7085/(61) 3107-6707. E-mail: fercoepsi@yahoo.com.br

Jairo Eduardo Borges-Andrade, doutor em Sistemas Instrucionais pela Florida State University, Estágios Senior no IFPRI (Washington, DC), University of Sheffield/University of Groningen, ISCTE-IUL, é professor Titular na Universidade de Brasília. Email: jairo@unb.br 